\title{
Selective Histone Deacetylase 6
} Inhibitor 23BB Alleviated Rhabdomyolysis-Induced Acute Kidney Injury by Regulating Endoplasmic Reticulum Stress and Apoptosis

\section{OPEN ACCESS}

Edited by:

Alfonso Pompella,

Università degli Studi di Pisa, Italy

Reviewed by:

Md Abdul Hye Khan,

Medical College of Wisconsin,

United States

Sabata Pierno,

Università degli Studi di Bari Aldo

Moro, Italy

${ }^{*}$ Correspondence:

Liang Ma

liang_m@scu.edu.cn

Ping Fu

fupinghx@163.com

†These authors have contributed equally to this work.

Specialty section:

This article was submitted to Experimental Pharmacology and Drug

Discovery,

a section of the journal

Frontiers in Pharmacology

Received: 11 January 2018 Accepted: 12 March 2018

Published: 26 March 2018

Citation:

Feng $Y$, Huang $R$, Guo $F$, Liang $Y$, Xiang J, Lei S, Shi M, Li L, Liu J, Feng Y, Ma L and Fu P (2018) Selective Histone Deacetylase 6 Inhibitor 23BB Alleviated Rhabdomyolysis-Induced Acute Kidney Injury by Regulating Endoplasmic Reticulum Stress and Apoptosis.

Front. Pharmacol. 9:274. doi: 10.3389/fphar.2018.00274
Yuying Feng ${ }^{1 \dagger}$, Rongshuang Huang ${ }^{1+}$, Fan Guo ${ }^{2}$, Yan Liang ${ }^{2}$, Jin Xiang ${ }^{3}$, Song Lei ${ }^{4}$, Min Shi ${ }^{1}$, Lingzhi Li ${ }^{1}$, Jing Liu' ${ }^{1}$, Yanhuan Feng ${ }^{1}$, Liang Ma ${ }^{1 *}$ and Ping Fu ${ }^{1 *}$

1 Division of Nephrology, Kidney Research Institute, West China Hospital of Sichuan University, Chengdu, China, ${ }^{2}$ Core Facility of West China Hospital, West China Hospital of Sichuan University, Chengdu, China, ${ }^{3}$ Laboratory of Clinical Pharmacology, West China Hospital of Sichuan University, Chengdu, China, ${ }^{4}$ Department of Pathology, West China Hospital of Sichuan University, Chengdu, China

Histone deacetylase 6 (HDAC6) contributed to the pathogenesis of rhabdomyolysisinduced acute kidney injury (AKI) and selective inhibition of HDAC6 activity may be a promising strategy for the treatment of AKI. Compound 23BB as a highly selective HDAC6 inhibitor was designed, synthesized by our lab and exhibited therapeutic potential in various cancer models with good safety. However, it remained unknown whether 23BB as a drug candidate could offer renal protective effect against rhabdomyolysis-induced AKI. In the present study, we investigated the effect of 23BB in a murine model of glycerol (GL) injection-induced rhabdomyolysis. Following GL injection, the mice developed severe $\mathrm{AKI}$ as indicated by acute renal dysfunction and histologic changes, accompanied by increased HDAC6 expression in the cytoplasm of tubular epithelial cells. Pharmacological inhibition of HDAC6 by 23BB pretreatment significantly reduced serum creatinine and serum blood urea nitrogen (BUN) levels as well as attenuated renal tubular damage in GL-injured kidneys. HDAC6 inhibition also resulted in reduced TdT-mediated dUTP nick-end labeling (TUNEL)-positive tubular cells, suppressed BAX, BAK, cleaved caspase-3 levels, and preserved Bcl-2 expression, indicating that 23BB exerted potent renoprotective effects by the regulation of tubular cell apoptosis. Moreover, GL-induced kidney injury triggered multiple signal mediators of endoplasmic reticulum (ER) stress including GRP78, CHOP, IRE1 $\alpha$, p-elF2 $\alpha$, ATF4, XBP1, p-JNK, and caspase-12. Oral administration of 23BB improved above-mentioned responses in injured kidney tissues and suggested that 23BB modulated tubular cell apoptosis via the inactivation of ER stress. Overall, these data highlighted that renal protection of novel HDAC6 inhibitor 23BB is substantiated by the reduction of ER stress-mediated apoptosis in tubular epithelial cells of rhabdomyolysis-induced AKI.

Keywords: rhabdomyolysis, acute kidney injury, histone deacetylase 6 inhibitor, endoplasmic reticulum stress, apoptosis 


\section{INTRODUCTION}

Acute kidney injury (AKI), characterized by a rapid decline of the glomerular filtration rate, is a serious clinical problem correlated with an aggressive disease course, high rates of mortality and increased risk of chronic kidney diseases (CKD) (Venkatachalam et al., 2015). Rhabdomyolysis, accounts for $15 \%$ of AKI cases (Chatzizisis et al., 2008), could be induced by different conditions including metabolism disorders, trauma, drugs, and toxins (e.g., statins, alcohol, and cocaine), infections, etc. (Sever et al., 2006; Bosch et al., 2009).

Although the detailed mechanisms have not been fully comprehended, it has been well-established that endoplasmic reticulum (ER) stress-mediated apoptosis of tubular epithelium cells played crucial roles in rhabdomyolysis-induced AKI (Feng et al., 2016). Organelle-mediated stress, particularly ER stress, has recently emerged as a major pathophysiological paradigm underlying apoptosis. The presence of misfolded proteins and other stresses lead to the activation of an adaptive program by the ER, known as the unfolded protein response (UPR), to restore protein-folding homeostasis (Walter and Ron, 2011). Initiation of the canonical UPR engages three distinct signaling branches, which are mediated by pancreatic ER kinase (PERK), activating transcription factor-6 (ATF6) and inositol-requiring transmembrane kinase/endonuclease-1 (IRE-1) (Gardner and Walter, 2011; Wang and Kaufman, 2016). The UPR is also linked to the activation of stress kinases such as the c-Jun $\mathrm{N}$-terminal kinase (JNK) and splicing of X-box binding protein 1 (XBP1) (Calfon et al., 2002; Kim et al., 2006). The combined action of these pathways results in the inhibition of protein translation, stimulation of protein degradation and production of chaperone proteins, triggering either recovery of ER function or cell death (Kim et al., 2006).

Many cellular functions, including apoptosis, are regulated by the acetylation of histone and non-histone proteins (Jazirehi, 2010; Liu and Zhuang, 2015). An acetyl group can be added to a lysine residue by histone acetyl transferases (HATs) or be removed by histone deacetylases (HDACs). HDACs are classified into four classes based on the structure and homology: class I HDACs (HDAC1, 2, 3, and 8); class II HDACs (HDAC4, 5, 6, 7, 9, and 10); class III HDACs (SIRT1-7); and class IV (HDAC11) (Ropero and Esteller, 2007). Among the 11 isoforms of HDACs, HDAC6 activation has been reported to be involved in the pathogenesis of rhabdomyolysis-induced AKI for the contribution to renal tubular cell apoptosis, inflammatory response, macrophage infiltration and oxidative stress (Liu and Zhuang, 2015; Shi et al., 2017). Therefore, selective inhibition of HDAC6 activity may be a promising strategy for the treatment of AKI.

In our previous study, highly selective HDAC6 inhibitor 23BB has been designed, synthesized and evaluated for anti-tumor activity and safety in both solid and hematologic tumor models (Figure 1) (Yang et al., 2016). It demonstrated low nanomolar anti-proliferative effects against panel of cancer cell lines and more effectively inhibited the tumor growth than SAHA even at a fourfold reduced dose or ACY-1215 at the same dose. However, the therapeutic potential of $23 \mathrm{BB}$ as a drug candidate in rhabdomyolysis-induced AKI had not been explored. In the study, we aimed to investigate whether $23 \mathrm{BB}$ protected against rhabdomyolysis-induced AKI by selectively inhibiting HDAC6 and to determine the involved mechanisms.

\section{MATERIALS AND METHODS}

\section{Animals}

This study adheres to the "Principles of Laboratory Animal Care" (National Institutes of Health publication 85-23, revised 1985) that seeks to minimize both the number of animals used and any suffering that they might experience, and is conducted in accordance with a protocol approved by Animal Care and Use Ethics Committee of Sichuan University in China (IACUC number: 2017080A). Twenty-four healthy 8-week male C57BL/6 mice were housed in a controlled environment (constant temperature at $20 \pm 2^{\circ} \mathrm{C}$ and humidity at $50-60 \%$ with a 12-h light and 12-h dark cycle) and had free access to standard laboratory food and water. The mice were housed for 1 week of adaptation before further research.

\section{Glycerol-Induced AKI Model and 23BB Administration}

The 24 mice were randomly divided into three groups $(n=8$ per group): the control group, the glycerol group, and the $23 \mathrm{BB}$ group. The mice in the glycerol group were injected with 50\% glycerol dissolved in $0.9 \%$ normal saline ( $10 \mu \mathrm{L} / \mathrm{g}$ body weight) at bilateral back limbs to elicits rhabdomyolysis-induced AKI model. The mice in the control group received an intramuscular injection of the same volume of vehicle (saline). As for the $23 \mathrm{BB}$ group, 23BB was dissolved in PEG and diluted in $0.9 \%$ normal saline to be orally administered at a dose of $40 \mathrm{mg} / \mathrm{kg} / \mathrm{d}$ for 3 days before the glycerol injection.

The mice were sacrificed at $24 \mathrm{~h}$ after the glycerol injection. Blood sample was collected and stored at $-80^{\circ} \mathrm{C}$. The upper half of the left kidney was quickly removed and fixed in $10 \%$ phosphate buffered formalin for PAS staining, IHC and TUNEL assay. The lower half of the left kidney was fixed in $2.5 \%$ glutaraldehyde for $2 \mathrm{~h}$ at $4^{\circ} \mathrm{C}$ and processed for transmission electron microscope. The right kidney was quickly removed and frozen in liquid nitrogen, then stored at $-80^{\circ} \mathrm{C}$ until processed for immunoblot analysis and immunofluorescence staining.

\section{Serum Analysis}

Serum creatinine $(\mathrm{sCr})$, blood urea nitrogen $(\mathrm{BUN})$ and serum creatine kinase (CK) levels were evaluated by high performance liquid chromatography (HPLC) conducted by the Institute of Drug Clinical Trial and the GCP center of West China Hospital. The AKI model was considered established when the level of serum creatinine of the treatment group rose up to 2 times of their control littermates.

\section{Histologic Examination}

The upper half of the left kidney, fixed in $10 \%$ phosphate buffered formalin, was dehydrated in a graded series of alcohol 


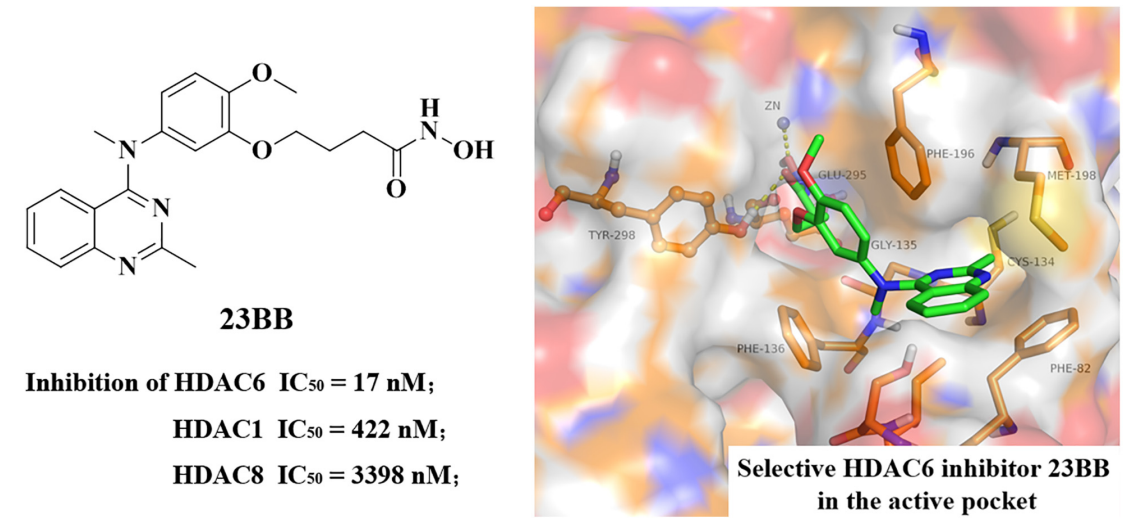

FIGURE 1 | Chemical structure of 23BB and homology model of 23BB docked into HDAC6.

concentrations and embedded in paraffin. Kidney blocks were cut into $2 \mu \mathrm{m}$ sections and then subject to PAS staining for morphologic analysis and TUNEL staining for cell apoptosis.

Periodic acid-Schiff stained tissue sections were viewed by light microscopy at magnifications of $\times 200$ or $\times 400$. For semiquantitative analysis of morphological changes, two sections were randomly selected from each sample of at least 3 for every group and 10 fields were randomly selected at a magnification of $\times 200$ from each section in periodic acid-Schiff staining. Histopathological changes were evaluated by the percentage of injured/damaged renal tubules, as indicated by tubular lysis, dilation, disruption, and cast formation. Tissue damages were scored on a scale of $0-4$, with $0,1,2,3$, and 4 corresponding to $0 \%,<25 \%, 26 \%-50 \%, 51 \%-75 \%$, $\geq 76 \%$ of injured/damaged renal tubules, respectively.

TUNEL stained tissue sections were viewed by light microscopy at magnifications of $\times 200$ or $\times 400$. The sections were stained according to the manufacturer's protocol (Roche Molecular System, Branchburg, NJ, United States). Positive cells were counted at magnification of $\times 200$, and at least 10 fields per section for each sample were examined $(n=8)$.

\section{Electron Microscopy}

After being fixed in cold $2.5 \%$ glutaraldehyde for $2 \mathrm{~h}$ at $4^{\circ} \mathrm{C}$, kidney tissues were washed with phosphate-buffered saline (PBS) $(0.2 \mathrm{~mol} / \mathrm{L}, \mathrm{pH} 7.4)$ for $2 \mathrm{~h}$, fixed with $1 \%$ osmic acid for $2 \mathrm{~h}$, and then washed six times with PBS for 10 min per wash. The samples were dehydrated with ethanol and cleaned with epoxypropane. They were embedded in EPON 812 overnight at room temperature. Ultrathin sections $(40-60 \mathrm{~nm})$ were cut (EM UC61rt, Leica) and stained with uranyl acetate/lead citrate. These sections were subsequently visualized using a transmission electron microscope (H-7650, Hitachi).

\section{Immunoblot Analysis}

Mouse kidney cortexes were dissected and homogenized in radio immune precipitation (RIPA) lysis buffer (P0013B, Beyotime Biotechnology, China). After centrifugation at 13,000 rpm for $15 \mathrm{~min}$ at $4^{\circ} \mathrm{C}$, the supernatant was collected, and protein concentrations were determined using a bicinchoninic acid (BCA) Protein Assay Kit (Beyotime Institute of Biotechnology). Bovine serum albumin was used as the standard. Equal amounts of protein lysate were loaded directly on 10-12\% SDS-PAGE, transferred onto polyvinylidene difluoride (PVDF) Membrane for Protein Blotting $(0.2 \mu \mathrm{m}$, Bio-Rad Laboratories, Inc.). The membranes were blocked with 5\% non-fat dry milk (w/v) in Tris-buffered saline with $0.1 \%$ Tween-20 (TBS-T) for $1 \mathrm{~h}$ at room temperature and then incubated with indicated primary antibodies overnight at $4^{\circ} \mathrm{C}$. After being rinsed thrice with TBS-T at 5-min intervals, the membranes were incubated with horseradish peroxidase-labeled goat anti-rabbit IgG (1:2000 dilution; Biosynthesis Biotechnology Co., Ltd., Beijing, China) or goat anti-mouse IgG (1:2000 dilution; Biosynthesis Biotechnology Co., Ltd., Beijing, China) for $1 \mathrm{~h}$. Immunoblots were visualized using the Immobilon Western Chemiluminescent HRP Substrate (Millipore Corporation, Billerica, MA, United States) with Bio-Rad Chemi Doc MP. All immunoblot analysis data are from experiments performed in triplicate. Densitometry analysis was performed using ImageJ6.0 software (National Institutes of Health, Bethesda, MD, United States).

\section{Immunofluorescence Staining}

Renal tissues were fixed in $4.5 \%$ buffered formalin, dehydrated, and embedded in paraffin. For immunofluorescent staining, the tissue sections were rehydrated and blocked with PBS+5\% normal goat serum for $1 \mathrm{~h}$, after which they were labeled with indicated antibodies in a humidified chamber overnight at $-4^{\circ} \mathrm{C}$. The sections were exposed to Cy5 red-labeled or FITC green-labeled secondary antibodies (Jackson ImmunoResearch Inc., West Grove, PA, United States). The nuclei were counterstained with DAPI (1:500, Life Technologies Corporation, OR, United States). the images were captured with AxioCam HRc digital camera (Carl Zeiss).

\section{Immunohistochemistry}

After fixation in $10 \%$ phosphate buffered formalin overnight, the fixed kidneys were dehydrated through a graded series of 

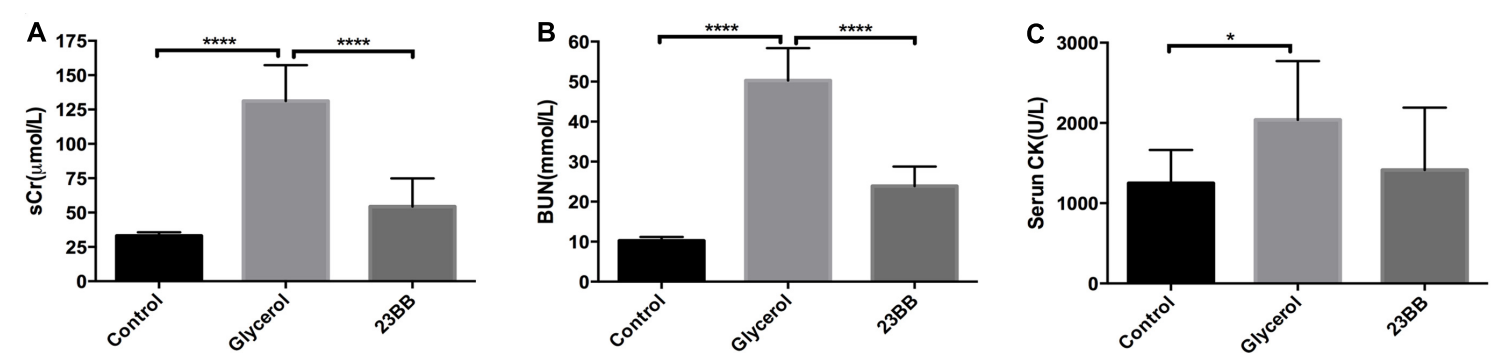

D
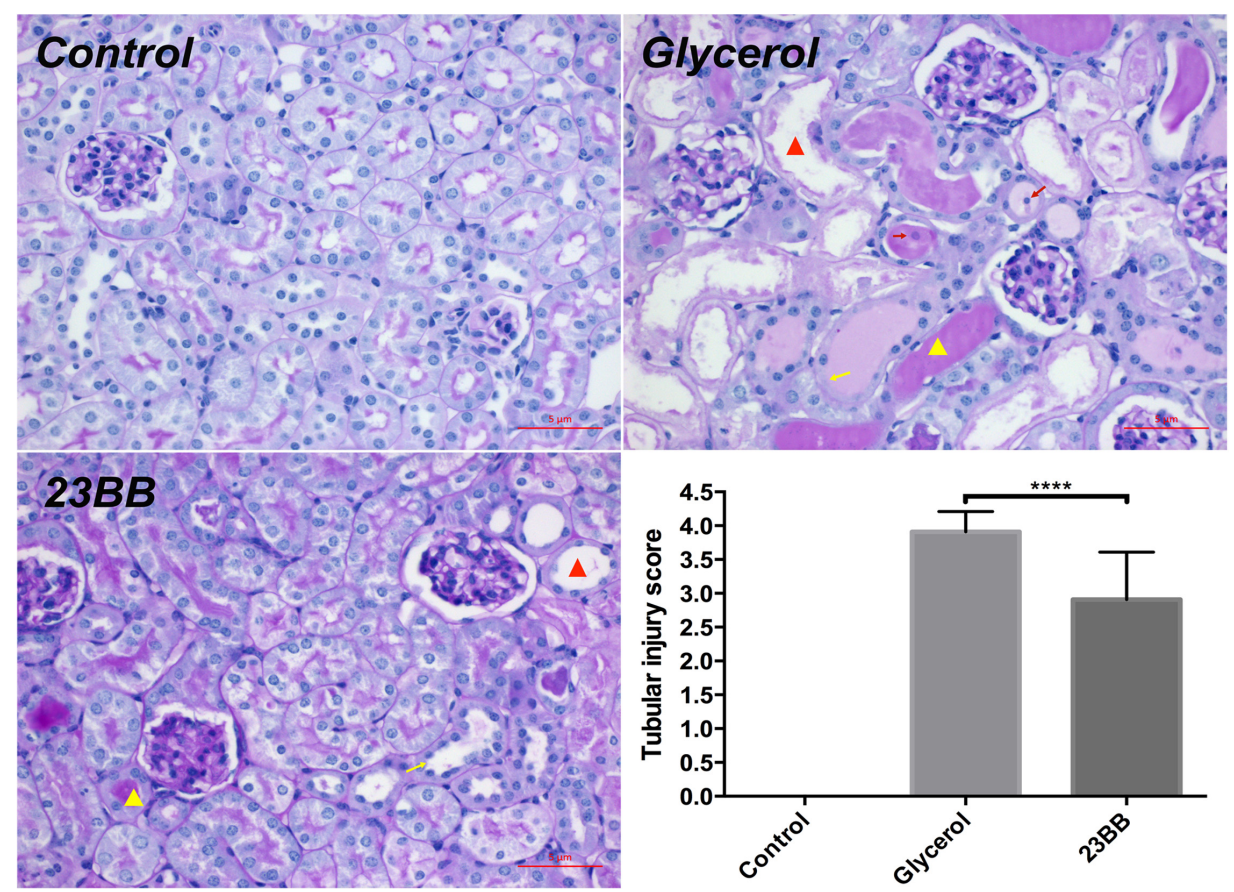

FIGURE 2 | Inhibition of HDAC6 with 23BB alleviates glycerol-induced AKI. (A) Serum creatinine (sCr). (B) Serum blood urea nitrogen (BUN). (C) Serum creatine kinase (CK). (D) Photomicrographs (X400) illustrate periodic acid-Schiff (PAS) staining of the kidney tissues (red triangle: tubular dilatation; red arrow: necrosis; yellow triangle: cast formation; yellow arrow: loss of brush border). Histologic changes were scored based on the scale described in the Section "Materials and Methods." Data are represented as the means $\pm \mathrm{SE}\left(n=6\right.$ for each group). $* * * * P<0.0001 ;{ }^{*} P<0.05$

ethanol, embedded in paraffin, sectioned ( $5 \mu \mathrm{m})$, and mounted on glass slides. The slides were blocked with $2.5 \%$ normal goat serum and incubated with primary antibodies at $4^{\circ} \mathrm{C}$. The slides were washed thrice in PBS, and VECTASTAIN ABC Kit (Vector, Burlingame, CA, United States) was used for staining following the manufacturer's instruction. The sections were counterstained with hematoxylin. Images were captured using an AxioCam HRc digital camera (Carl Zeiss).

\section{Primary Antibodies}

Anti-HDAC6 (sc11420, Santa Cruz), anti- acetyl-histone H3 (9649, Cell Signaling Technology), anti-BAX (ab32503, Abcam), anti-Bcl-2 (ab3214, Abcam), anti-cleaved caspase-3 (9664, Cell Signaling Technology), anti-phospho-JNK (4668, Cell Signaling Technology), anti-JNK (ab208035, Abcam), anti-phospho-eIF2 $\alpha$ (3398, Cell Signaling Technology), anti-eIF2 $\alpha$ (5324, Cell Signaling Technology),anti-phospho-PERK (sc32577, Santa Cruz) anti- GRP78 (ab108613, Abcam), anti- IRE1 $\alpha$ (ab48187,
Abcam), anti-CHOP (2895, Cell Signaling Technology), antiATF4 (11815, Cell Signaling Technology), anti- XBP1 (ab37152, Abcam), anti-caspase-12 (ab62484, Abcam).

\section{Statistical Analysis}

All experiments were performed in triplicate unless otherwise stated. Data are presented as mean \pm SEM. Data were subjected to analysis of variance (ANOVA) followed by Tukey's post hoc test, and unpaired Student's $t$-test for comparison between two groups. $P$-values $<0.05$ were considered statistically significant.

\section{RESULTS}

\section{Novel HDAC6 Inhibitor 23BB Alleviated Rhabdomyolysis-Induced AKI}

To determine whether $23 \mathrm{BB}$ may have a renal protective effect by targeting HDAC6, we examined the renal function and 

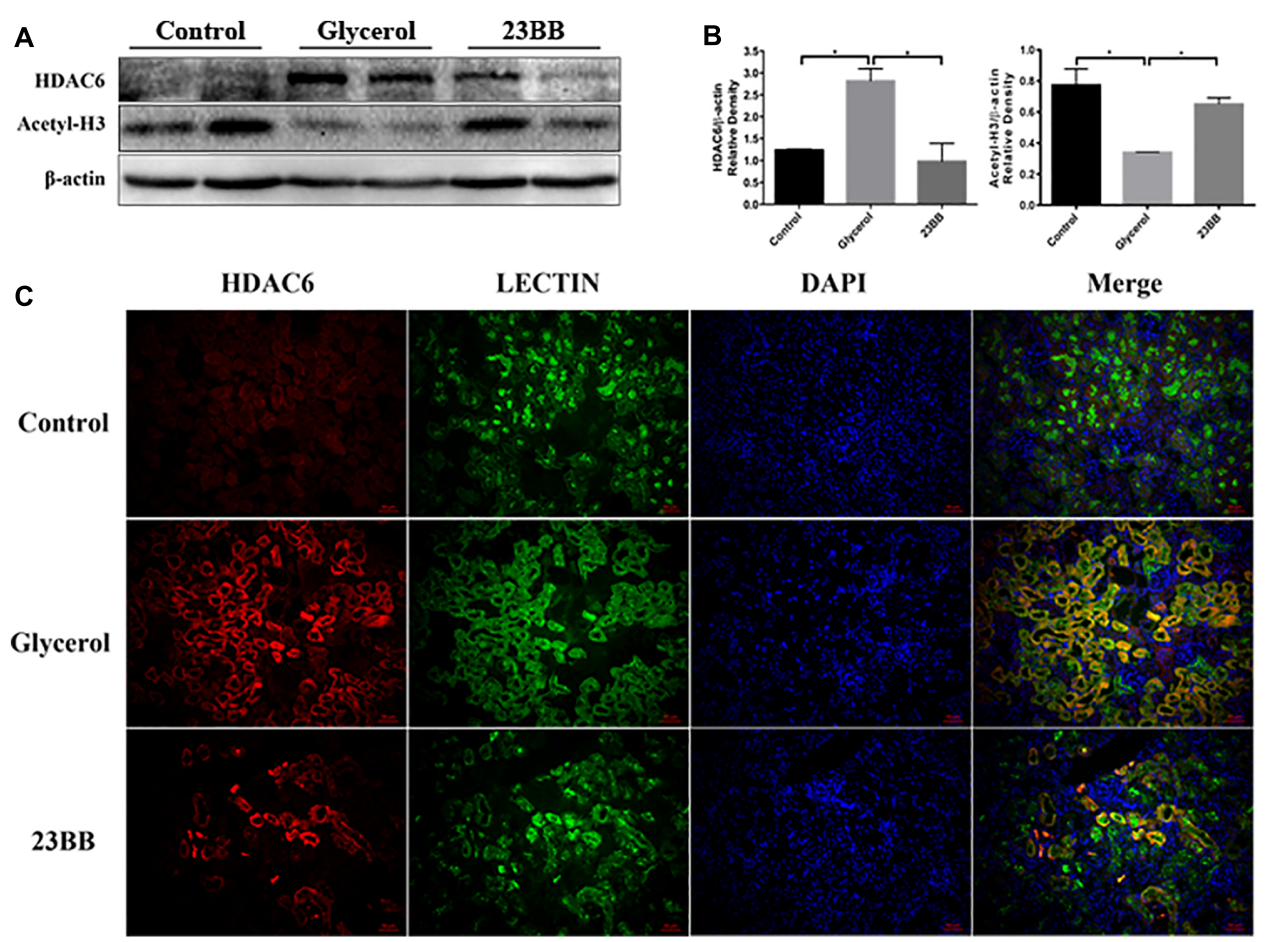

FIGURE 3 | 23BB inhibits the expression of HDAC6 and enhances the acetylation of histone H3. (A) The kidney tissue lysates were subjected to immunoblot analysis with indicated antibodies against HDAC6 and acetylated histone H3. (B) Expressions of HDAC6 and acetylated histone H3 were quantified by densitometry and normalized with $\beta$-actin. Data are represented as the means $\pm \mathrm{SE}$ ( $n=6$ for each group). ${ }^{*}<0.05$. (C) Immunofluorescence staining of HDAC6 and Lectin in the kidney tissue sections. Lectin was used as a marker of tubular epithelial cells.

pathological changes in a mouse model of rhabdomyolysisinduced AKI. As demonstrated in Figure 2, serum creatinine $(\mathrm{sCr})$, blood urea nitrogen $(\mathrm{BUN})$ and serum creatine kinase (CK) were markedly elevated at $24 \mathrm{~h}$ after GL injection. Pretreatment of $23 \mathrm{BB}$ at a dose of $40 \mathrm{mg} / \mathrm{kg} / \mathrm{d}$ for $3 \mathrm{~d}$ significantly improved acute renal dysfunction without influencing the level of serum CK. Consistent with improved kidney function by 23BB administration, a PAS-stained section showed less tubular dilatation, swelling, necrosis, cast formation and preservation of a brush border in the GL+23BB administered group as compared to that of GL group, which was indicated by the quantification of the kidney injury score (Figure 2D).

Taken together, these data suggested that compound $23 \mathrm{BB}$ protected against rhabdomyolysis-induced AKI via the inhibition of HDAC6 activity.

\section{HDAC6 Inhibitor 23BB Enhanced the Acetylation of Histone H3 in the Kidney of Rhabdomyolysis-Induced AKI}

Inhibition of HDAC can be reflected by the increased expression of acetyl histone H3 (Shi et al., 2017). To understand the inhibitory effect of 23BB on HDAC6 activity, we examined the expression of acetyl histone H3 and HDAC6 by immunoblot analysis. As exhibited in Figures 3A,B, the increased expression of HDAC6 was detected in GL-injected group, which was suppressed by 23BB pretreatment. Meanwhile, GL group showed a remarkable decrease of acetyl histone $\mathrm{H} 3$ level compared to that of control group, and the change was restored by $23 \mathrm{BB}$ administration.

Furtherly, to investigate whether HDAC6 was expressed in renal tubular epithelia cells, kidney tissues were stained by HDAC6 and Lotus tetragonolobus lectin (Lectin), a proximal epithelial cell marker. As demonstrated in Figure 3C, we found that HDAC6 was minimally expressed in the control group, but remarkably upregulated in GL group and was co-stained with Lectin. Oral administration of $23 \mathrm{BB}$ significantly suppressed the HDAC6 expression, which was consistent with results of immunoblot analysis. These findings indicated that glycerol induced the upregulation of HDAC6 in tubular epithelial cells, and that 23BB largely diminished this response.

\section{Inhibition of HDAC6 Activity Decreased Renal Tubular Cell Apoptosis in Rhabdomyolysis-Induced AKI}

Renal tubular cell apoptosis was a prominent feature in the development of rhabdomyolysis-induced AKI (Tang et al., 2013). To examine the role of HDAC6 in the model, we first analyzed the tubular cell apoptosis by TdT-mediated dUTP nick-end labeling (TUNEL) staining. As expected, large number of TUNEL-positive renal tubular cells were observed 

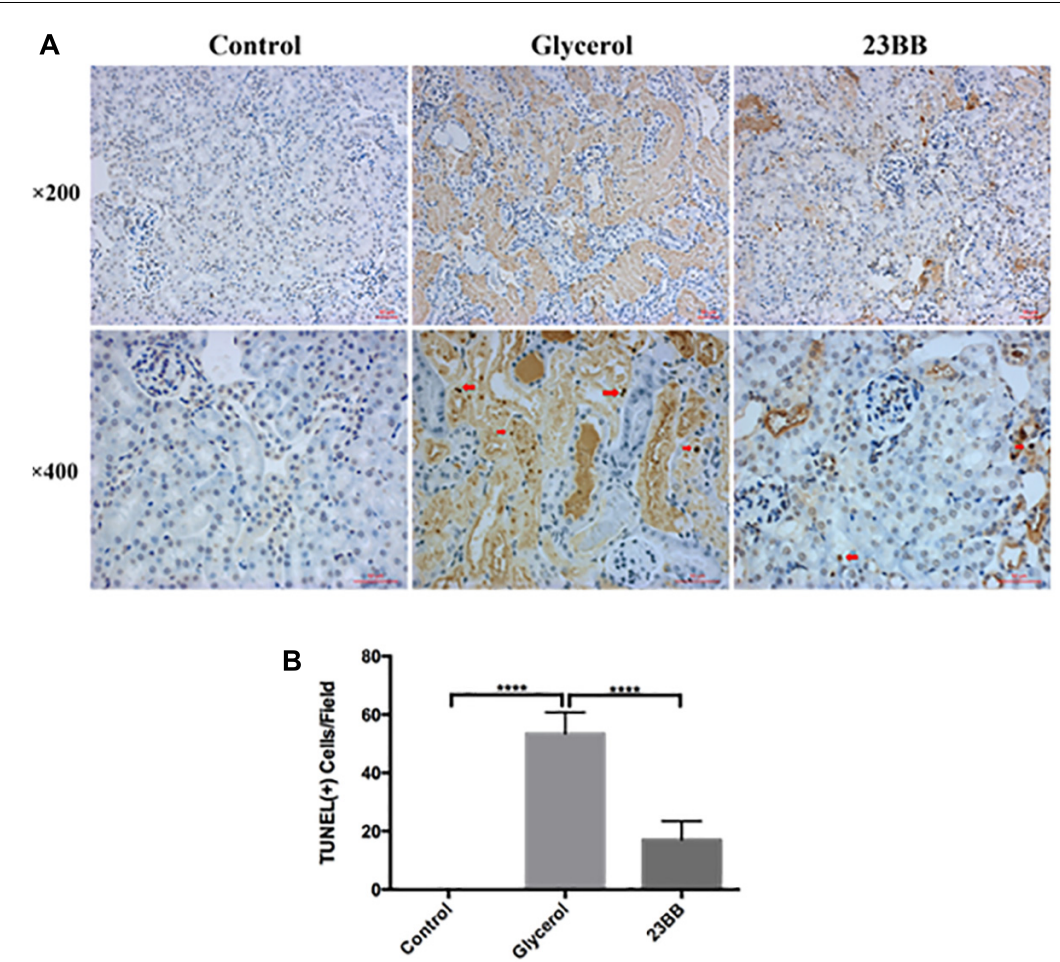

FIGURE 4 | Inhibition of HDAC6 activity decreased renal tubular cell apoptosis. (A) TUNEL staining of kidney tissue sections were collected at $\times 200$ and $\times 400$ magnifications (red arrow: TUNEL positive cells). (B) Positive TUNEL staining cells were counted and expressed as means \pm SE. $* * * * P<0.0001$.

in the injured kidney after GL injection. In comparison, 23BB administration diminished TUNEL-positive tubular cells (Figure 4).

Caspase-3 was the key executioner which ultimately modified proteins responsible for cell apoptosis (Savitskaya and Onishchenko, 2015). B-cell lymphoma 2 (Bcl-2) family consisted of a group of essential regulatory factors in cell apoptosis. Bcl-2 was a representative group I member which was anti-apoptotic, while Bcl-2-associated $\mathrm{X}$ (BAX) and $\mathrm{Bcl}-2$ homologous antagonist/killer (BAK) were representative group II members which were apoptotic (Cory and Adams, 2002; Youle and Strasser, 2008). In the study, we further examined the expression of BAX, BAK, Bcl-2 and cleaved caspase- 3 in the kidney after GL injection with or without $23 \mathrm{BB}$ administration by immunoblot analysis (Figure 5). Consistent with TUNEL staining results, GL injection induced tubular cell apoptosis, as assessed by the up-regulation of BAX, BAK and down-regulation of Bcl-2, besides boosting cleaved caspase-3 levels. However, $23 \mathrm{BB}$ administration led to marked renal protection against GL-induced apoptosis, as judged by restored the expressions of apoptosis-related markers. Immunofluorescence staining or IHC of the proteins were also conducted and the expressions in renal tubular cells were consistent with the results of immunoblot analysis.

Collectively, these data demonstrated that the selective inhibitory effect of 23BB on HDAC6 activity contributed to ameliorated renal tubular cell apoptosis in rhabdomyolysisinduced AKI.

\section{Pharmacological HDAC6 Inhibition Suppressed ER Stress and UPR in Rhabdomyolysis-Induced AKI}

By transmission electron microscopy (Figure 6), in the GL group, we observed large amount of swelling ER in the cytoplasm of renal tubular cells, which was not detected in the control group. Other ultrastructural changes including mitochondrial swelling and loss of brush border were also observed in GL group (Supplementary Figure 1). The administration of 23BB dramatically reduced swelling ER, while the mitochondrial swelling and the brush border loss were still detected. We hypothesized that 23BB ameliorated apoptosis through the suppression of ER stress.

To validate the hypothesis, we first conducted immunoblot analysis of ER stress related proteins including GRP78, IRE1 $\alpha$, p-eIF2 $\alpha$, ATF4, CHOP, p-JNK, caspase-12, and XBP1. As indicated in Figures 7, 8, GL injection induced the upregulation of ER stress related proteins in the injured kidney, which was significantly suppressed by $23 \mathrm{BB}$ administration. Furthermore, we performed IHC staining of GRP78 and immunofluorescence staining of IRE1 $\alpha$, ATF4, CHOP and XBP1. We found these proteins were minimally expressed in the control group, but remarkably upregulated in glycerol group, predominantly located in the renal tubules. Pretreatment of HDAC6 inhibitor 23BB significantly suppressed ER stress related proteins. Thus, these findings highlighted that $23 \mathrm{BB}$ administration inhibited the activation of ER stress in glycerol-induced AKI. 

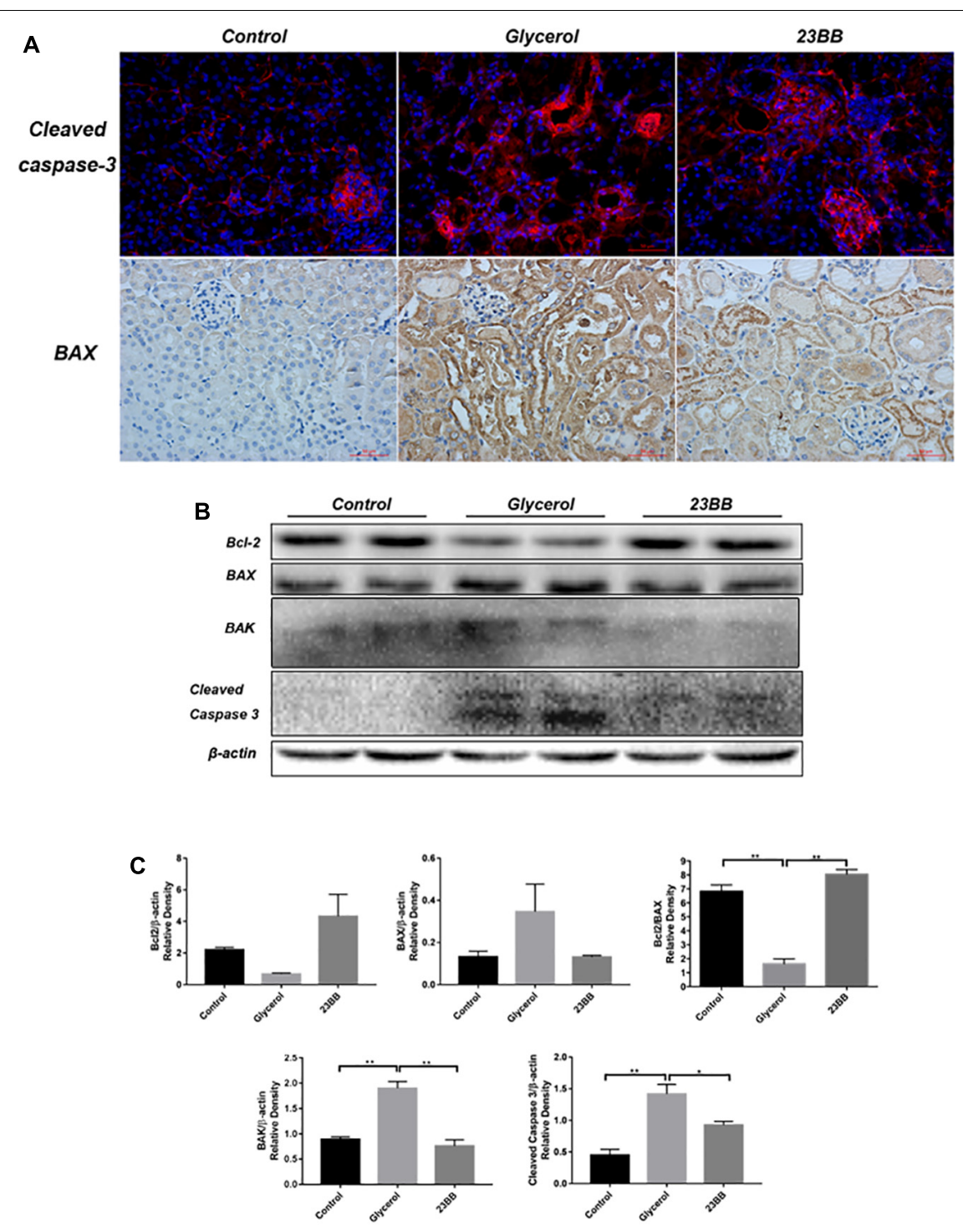

FIGURE 5 | 23BB inhibits the expression of apoptosis related proteins. (A) Immunofluorescence staining was performed to detect expression of cleaved caspase-3 in kidney tissue sections in each group, immunohistochemistry illustrated BAX-stained kidney sections in each group. (B) The kidney tissue lysates were subjected to immunoblot analysis with indicated antibodies against BAX, Bcl-2, BAK and cleaved caspase-3. (C) Expressions of BAX, Bcl-2, BAK and cleaved caspase-3 were quantified by densitometry and normalized with $\beta$-actin. Data are represented as the means \pm SE $\left(n=6\right.$ for each group). ${ }^{* *} P<0.01$; ${ }^{*} P<0.05$.

\section{DISCUSSION}

Previous studies have confirmed that HDAC6 contributed to the pathogenesis of rhabdomyolysis-induced AKI and selective inhibition might be a promising strategy for the treatment of AKI (Shi et al., 2017). Compound 23BB as a highly selective and potent HDAC6 inhibitor has been designed, synthesized and evaluated for anti-tumor activity in our lab (Yang et al., 2016). While in the study, we found that pharmacological inhibition of HDAC6 by 23BB improved acute renal dysfunction indicated by reduced $\mathrm{sCr}$ and $\mathrm{BUN}$ levels in a GL-induced AKI model. Pretreatment of $23 \mathrm{BB}$ alleviated renal tubular damage and attenuated ER stress-mediated apoptosis in the tubular epithelial cells of GL-injured kidney tissues. The involved mechanism of
23BB against rhabdomyolysis-induced AKI has been summarized in Figure 9. Our data demonstrated the protective effects of $23 \mathrm{BB}$ on rhabdomyolysis-induced AKI and provided new evidence regarding the potential therapeutic effects on AKI.

Apoptotic cell death is usually a response to cell microenvironment (Dang et al., 2015; Su et al., 2015). Apoptosis requires the activation of lethal molecules and the inactivation of prosurvival ones (Youle and Strasser, 2008). Apoptotic pathways are active in the tubular epithelium induced by caspase cascade activation, mitochondrial injury, and ER stress, etc. (Savitskaya and Onishchenko, 2015). Apoptosis also promotes renal epithelial cell loss that characterizes acute kidney diseases (Havasi and Borkan, 2011; Linkermann et al., 2014). Accumulating evidence has demonstrated that the caspase-3 


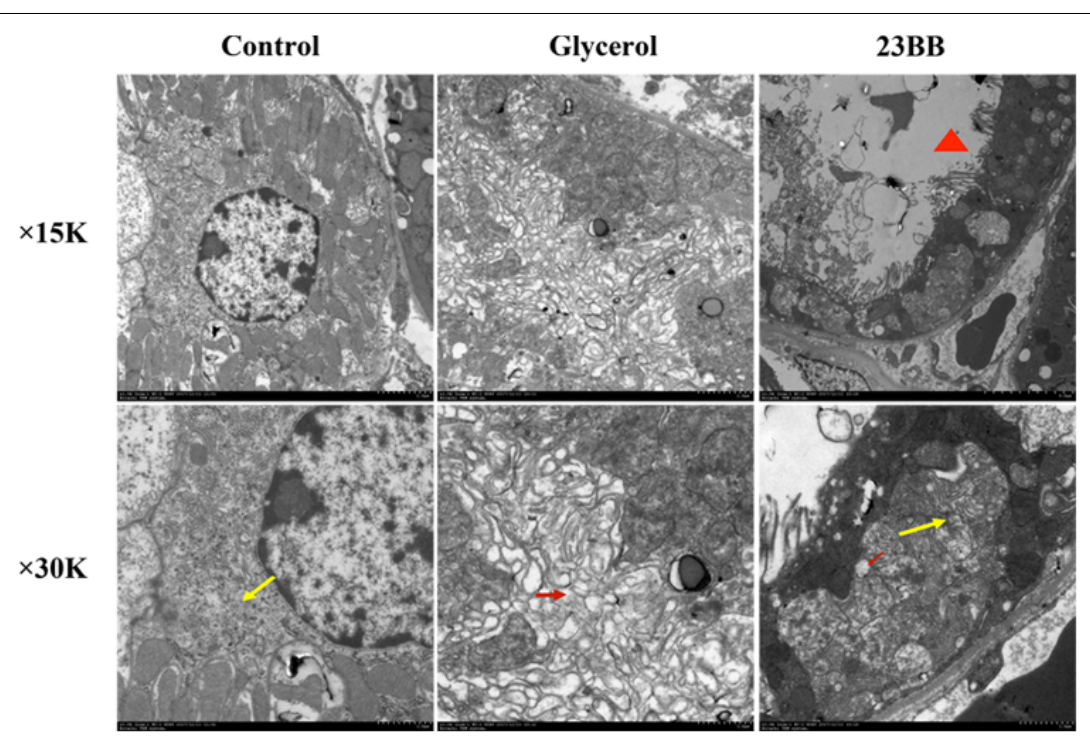

FIGURE 6 | 23BB administration attenuates endoplasmic reticulum expansion. Photomicrographs ( $\times 15 \mathrm{k}$ and $\times 30 \mathrm{k})$ collected by transmission electron microscope (yellow arrow: normal endoplasmic reticulum; red arrow: expansion of endoplasmic reticulum; red triangle: loss of brush border).

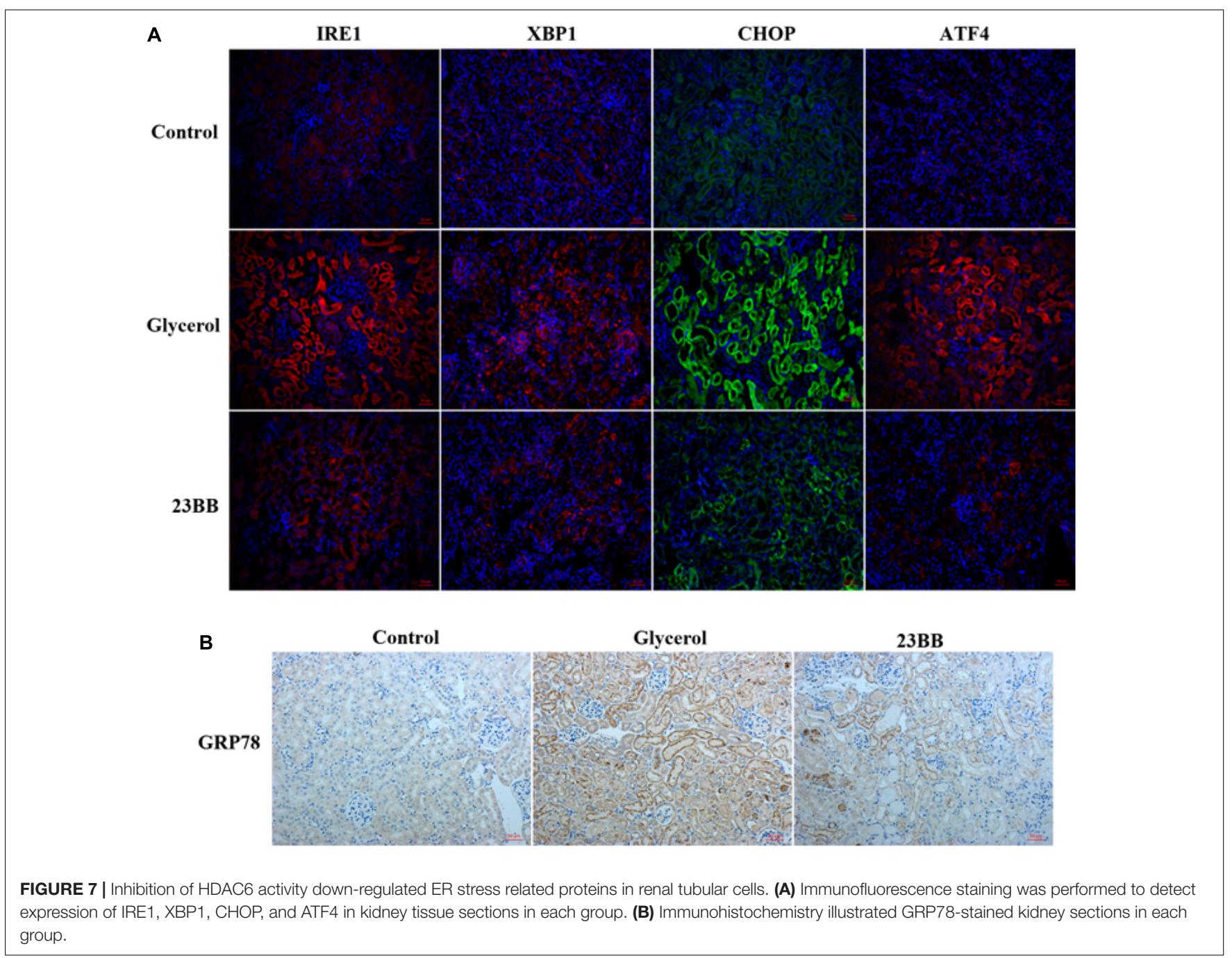



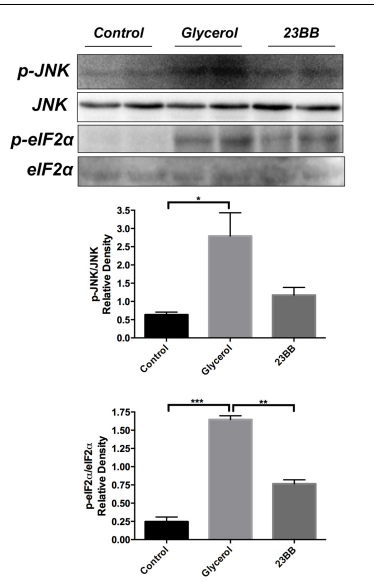
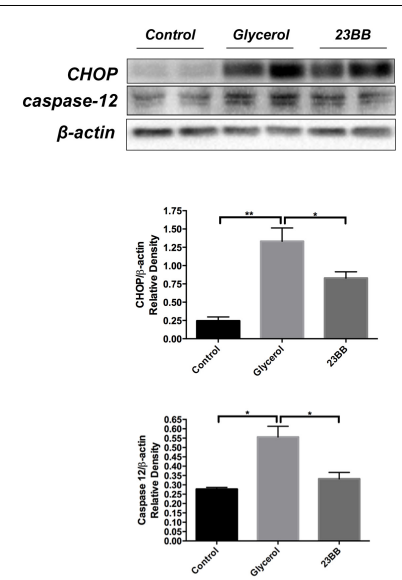
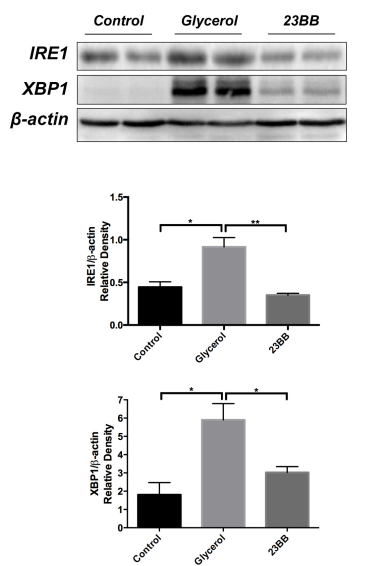
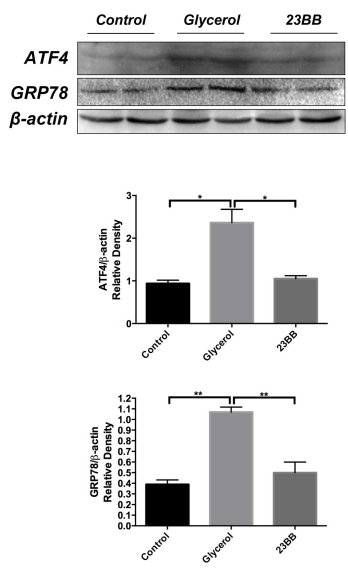

FIGURE 8 | 23BB administration suppressed expression level of ER stress proteins. The expression of GRP78, IRE1 $\alpha$, p-elF2 $\alpha$, ATF4, CHOP, p-JNK, caspase-12, and XBP1, as measured by immunoblot analysis, in kidney tissue sections in each group. The densitometry values of $p$-JNK were normalized with JNK. The densitometry values of $\mathrm{p}$-elF2 $\alpha$ were normalized with elF2 $\alpha$. The densitometry values of other proteins were normalized with $\beta$-actin. Data are represented as the means \pm SE ( $n=6$ for each group). ${ }^{*} P<0.05$.

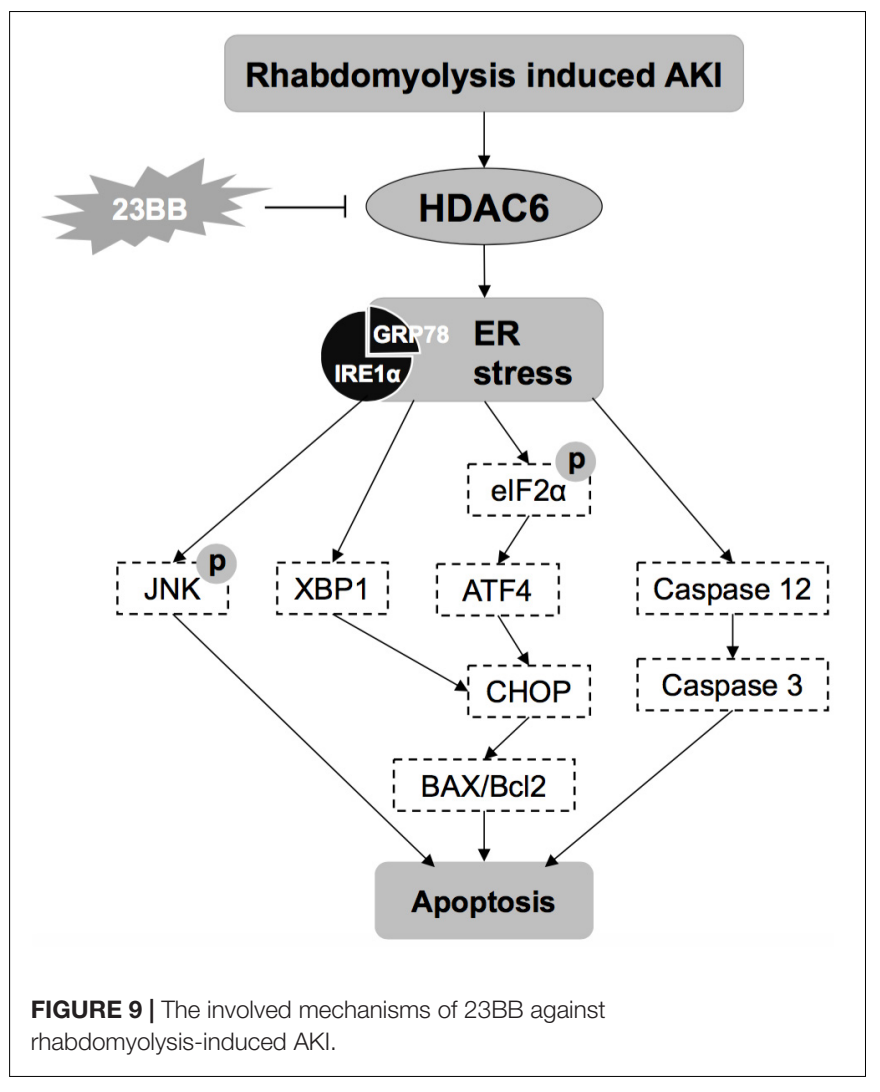

activation is the predominant mechanism responsible for the apoptosis of renal tubular cells in rhabdomyolysis-induced AKI (Linkermann et al., 2014; Feng et al., 2016). In our study, HDAC6 inhibition by $23 \mathrm{BB}$ blocked the cleavage of caspase- 3 in the injured kidney, suggesting that HDAC6 mediated the activation of caspase-3. Another crucial regulatory factor in apoptosis is the Bcl-2 family which could be divided into two major classes based on their functions and structures: group I proteins that are anti-apoptotic and group II proteins that are apoptotic (Cory and Adams, 2002; Youle and Strasser, 2008). Thus, the inhibition of group I proteins and/or the activation of group II proteins can successfully induce cell apoptosis. Herein, we evaluated the impact of $23 \mathrm{BB}$ on the level of anti-apoptotic $\mathrm{Bcl}-2$ and apoptotic BAX/BAK. We found that the blockage of HDAC6 by $23 \mathrm{BB}$ significantly suppressed BAX/BAK and preserved $\mathrm{Bcl}-2$ in the kidney tissues of rhabdomyolysis-induced AKI. Therefore, 23BB possessed inhibitory effects on tubular epithelial cell apoptosis in the pathogenesis of rhabdomyolysis-induced AKI.

Endoplasmic reticulum stress could be triggered by different stimulatory signals in AKI, including mutant protein aggregation, hypoxia, energy deprivation, and metabolic dysfunction (Taniguchi and Yoshida, 2015). Declined protein-folding capacity of ER leads to the accumulation of misfolded proteins, initiating ER stress. Overwhelming ER stress induces tubular cell apoptosis via three typical signal pathways, PERK-eIF2ATF4, IRE1-XBP1 and ATF6 pathway (Gardner and Walter, 2011). Under non-stressed conditions, glucose-regulated protein (GRP78) binds to its client proteins PERK, ATF6, and IRE1, preventing their signaling. However, when ER is overloaded, GRP78 binds to the unfolded proteins in the ER, freeing its client proteins, which then serves as the primary mediators of UPR signaling (Walter and Ron, 2011; Wang and Kaufman, 2016). UPR is a cellular quality control mechanism with two primary functions: first, to promote survival during ER stress by chaperoning proteins for re-folding and halting transcription and translation until homeostasis is restored; second, to signal CCAAT/enhancerbinding protein-homologous protein (CHOP) mediated apoptosis when homeostasis could not be re-established (Gorman et al., 2012). During UPR, PERK is released from its chaperone protein GRP78 to permit phosphorylation of eIF $2 \alpha$, leading to the activation of ATF4 and CHOP. Released 
IRE1 targets the downstream XBP1 and JNK, resulting in cell apoptosis (Calfon et al., 2002; Gardner and Walter, 2011). Apart from that, caspase-12, which is only expressed in rodents, is another major mediator of ER stress-mediated apoptosis that responds to ER stress and induces the caspase-3 cleavage to initiate apoptosis (Morishima et al., 2002).

Considerable evidence suggested some connection between HDAC inhibitors and ER stress in other disease models including lymphoma (Cosenza et al., 2017), breast cancer (Komatsu et al., 2013) and Duchenne muscular dystrophy (Consalvi et al., 2014). Previous study has reported that class I HDACs could localize ER, bind to GRP78, and selectively activate the UPR (Kahali et al., 2012). HDAC6, which primarily resides in the cytoplasm, contributed to the acetylation of ER-localized chaperone protein GRP78 (Li et al., 2016). In our study, we found that the inhibition of HDAC6 by 23BB significantly suppressed ER stress, as evidenced by the decreased expression of GRP78. The downstream UPR was also alleviated, as indicated by the diminished expression of IRE1 $\alpha$, XBP1, p-eIF2 $\alpha$, ATF4. Moreover, 23BB pretreatment down-regulated the expression of mediators of ER stressmediated apoptosis, including CHOP, p-JNK and caspase-12. In a nutshell, the protective effect of $23 \mathrm{BB}$ against tubular epithelial cell apoptosis is mediated via the suppression of ER stress.

Notably, the functional significance of HDACs in rhabdomyolysis-induced AKI remains controversial (Hui and Chiang, 2014). Given the fact that our study showed that HDAC6 inhibition by selective small molecule offered a protective effect on the injured kidney in the pathological condition, there were reports of blocking class I HDACs by MS-275 resulted in worsening renal dysfunction and enhancing cell apoptosis with caspase- 3 activation in the same AKI model (Tang et al., 2014). In support of our observations, administration of TA, another HDAC6 selective inhibitor, was reported to exert a similar protection in rhabdomyolysis-induced AKI model (Shi et al., 2017). This debate also applies to other AKI models. In cisplatin-induced AKI models, on one hand, there are studies showing that TSA suppresses cisplatin-induced tubular epithelial cell apoptosis through the suppression of p53 and restoration of CREB-mediated transcription (Arany et al., 2008; Dong et al., 2010). On the other hand, the proapoptotic effect of HDAC inhibitor SAHA and TSA were also reported in tubular epithelial cell apoptosis (Dong et al., 2008).

\section{REFERENCES}

Arany, I., Herbert, J., Herbert, Z., and Safirstein, R. L. (2008). Restoration of CREB function ameliorates cisplatin cytotoxicity in renal tubular cells. Am. J. Physiol. Renal Physiol. 294, F577-F581. doi: 10.1152/ajprenal.00487.2007

Bosch, X., Poch, E., and Grau, J. M. (2009). Rhabdomyolysis and acute kidney injury. N. Engl. J. Med. 361, 62-72. doi: 10.1056/NEJMra08 01327

Calfon, M., Zeng, H., Urano, F., Till, J. H., Hubbard, S. R., Harding, H. P., et al. (2002). IRE1 couples endoplasmic reticulum load to secretory capacity by processing the XBP-1 mRNA. Nature 415, 92-96. doi: 10.1038/415092a
A possible explanation is that different classes of HDACs may serve distinct roles in activating various signaling pathways that lead to the development of AKI. To what extent each isoform of HDAC contributes to AKI is not fully comprehended. Thus, these non-selective or partially selective HDAC inhibitors usually lead to undesirable biological responses that accelerate pathologic changes. HDAC6, which is primarily expressed in the cytoplasm, removes the acetyl group from lysine residues in many nonhistone substrates. In contrast to the lethal effect of HDAC1-3 genetic ablation, HDAC6-knocked out mice are viable and fertile (Ran et al., 2016). These results supported that HDAC6 selective inhibitor $23 \mathrm{BB}$ is a safe agent with fewer side effects than panHDAC inhibitors and may be a promising agent for the treatment of AKI.

\section{CONCLUSION}

In summary, our findings demonstrated that highly selective HDAC6 inhibitor 23BB protects against rhabdomyolysis-induced AKI via the regulation of ER stress-mediated apoptosis in tubular epithelial cells. Thus, compound $23 \mathrm{BB}$ as a promising drug candidate may be a novel strategy for the treatment of AKI.

\section{AUTHOR CONTRIBUTIONS}

LM and PF conceived and designed the experiments. YuF, RH, FG, YL, JX, SL, MS, and YaF performed the experiments. YuF, LL, $\mathrm{JL}$, and RH analyzed the data. YuF and LM wrote the paper. YuF, RH, FG, YL, JX, SL, MS, LL, JL, YaF, LM, and PF approved the final version of the manuscript.

\section{ACKNOWLEDGMENTS}

This work was supported by grant 81570668 from National Natural Science Foundation of China.

\section{SUPPLEMENTARY MATERIAL}

The Supplementary Material for this article can be found online at: https://www.frontiersin.org/articles/10.3389/fphar. 2018.00274/full\#supplementary-material

Chatzizisis, Y. S., Misirli, G., Hatzitolios, A. I., and Giannoglou, G. D. (2008). The syndrome of rhabdomyolysis: complications and treatment. Eur. J. Intern. Med. 19, 568-574. doi: 10.1016/j.ejim.2007.06.037

Consalvi, S., Saccone, V., and Mozzetta, C. (2014). Histone deacetylase inhibitors: a potential epigenetic treatment for Duchenne muscular dystrophy. Epigenomics 6, 547-560. doi: 10.2217/epi.14.36

Cory, S., and Adams, J. M. (2002). The Bcl2 family: regulators of the cellular life-or-death switch. Nat. Rev. Cancer 2, 647-656. doi: 10.1038/nr c883

Cosenza, M., Civallero, M., Marcheselli, L., Sacchi, S., and Pozzi, S. (2017). Ricolinostat, a selective HDAC6 inhibitor, shows anti-lymphoma cell activity 
alone and in combination with bendamustine. Apoptosis 22, 827-840. doi: 10.1007/s10495-017-1364-4

Dang, S., Yu, Z. M., Zhang, C. Y., Zheng, J., Li, K. L., Wu, Y., et al. (2015). Autophagy promotes apoptosis of mesenchymal stem cells under inflammatory microenvironment. Stem Cell Res. Ther. 6:247. doi: 10.1186/s13287-015-0245-4

Dong, G., Luo, J., Kumar, V., and Dong, Z. (2010). Inhibitors of histone deacetylases suppress cisplatin-induced p53 activation and apoptosis in renal tubular cells. Am. J. Physiol. Renal Physiol. 298, F293-F300. doi: 10.1152/ ajprenal.00410.2009

Dong, G., Wang, L., Wang, C. Y., Yang, T., Kumar, M. V., and Dong, Z. (2008). Induction of apoptosis in renal tubular cells by histone deacetylase inhibitors, a family of anticancer agents. J. Pharmacol. Exp. Ther. 325, 978-984. doi: 10.1124/ jpet.108.137398

Feng, Y. Y., Ma, L., Liu, L. F., Hong, G. H., Zhang, X. M., Guo, F., et al. (2016). Rhabdomyolysis induced AKI via the regulation of endoplasmic reticulum stress and oxidative stress in PTECs. RSC Adv. 6, 109639-109648. doi: 10.1039/ C6RA18865F

Gardner, B. M., and Walter, P. (2011). Unfolded proteins are Ire1-activating ligands that directly induce the unfolded protein response. Science 333, 1891-1894. doi: 10.1126/science. 1209126

Gorman, A. M., Healy, S. J., Jager, R., and Samali, A. (2012). Stress management at the ER: regulators of ER stress-induced apoptosis. Pharmacol. Ther. 134, 306-316. doi: 10.1016/j.pharmthera.2012.02.003

Havasi, A., and Borkan, S. C. (2011). Apoptosis and acute kidney injury. Kidney Int. 80, 29-40. doi: 10.1038/ki.2011.120

Hui, K. F., and Chiang, A. K. (2014). Combination of proteasome and class I HDAC inhibitors induces apoptosis of NPC cells through an HDAC6-independent ER stress-induced mechanism. Int. J. Cancer 135, 2950-2961. doi: 10.1002/ijc. 28924

Jazirehi, A. R. (2010). Regulation of apoptosis-associated genes by histone deacetylase inhibitors: implications in cancer therapy. Anticancer Drugs 21, 805-813. doi: 10.1097/CAD.0b013e32833dad91

Kahali, S., Sarcar, B., Prabhu, A., Seto, E., and Chinnaiyan, P. (2012). Class I histone deacetylases localize to the endoplasmic reticulum and modulate the unfolded protein response. FASEB J. 26, 2437-2445. doi: 10.1096/fj.11-19 3706

Kim, R., Emi, M., Tanabe, K., and Murakami, S. (2006). Role of the unfolded protein response in cell death. Apoptosis 11, 5-13. doi: 10.1007/s10495-005-3088-0

Komatsu, S., Moriya, S., Che, X. F., Yokoyama, T., Kohno, N., and Miyazawa, K. (2013). Combined treatment with SAHA, bortezomib, and clarithromycin for concomitant targeting of aggresome formation and intracellular proteolytic pathways enhances ER stress-mediated cell death in breast cancer cells. Biochem. Biophys. Res. Commun. 437, 41-47. doi: 10.1016/j.bbrc.2013.06.032

Li, Z., Zhuang, M., Zhang, L., Zheng, X., Yang, P., and Li, Z. (2016). Acetylation modification regulates GRP78 secretion in colon cancer cells. Sci. Rep. 6:30406. doi: 10.1038/srep30406

Linkermann, A., Chen, G., Dong, G., Kunzendorf, U., Krautwald, S., and Dong, Z. (2014). Regulated cell death in AKI. J. Am. Soc. Nephrol. 25, 2689-2701. doi: 10.1681/ASN.2014030262

Liu, N., and Zhuang, S. (2015). Treatment of chronic kidney diseases with histone deacetylase inhibitors. Front. Physiol. 6:121. doi: 10.3389/fphys.2015.00121

Morishima, N., Nakanishi, K., Takenouchi, H., Shibata, T., and Yasuhiko, Y. (2002). An endoplasmic reticulum stress-specific caspase cascade in apoptosis. Cytochrome c-independent activation of caspase-9 by caspase-12. J Biol Chem 277, 34287-34294. doi: 10.1074/jbc.M204973200
Ran, J., Yu, F., Qin, J., Zhang, Y., Yang, Y., Li, D., et al. (2016). Functional interplay between cylindromatosis and histone deacetylase 6 in ciliary homeostasis revealed by phenotypic analysis of double knockout mice. Oncotarget 7, 2752727537. doi: 10.18632/oncotarget.8374

Ropero, S., and Esteller, M. (2007). The role of histone deacetylases (HDACs) in human cancer. Mol. Oncol. 1, 19-25. doi: 10.1016/j.molonc.2007.01.001

Savitskaya, M. A., and Onishchenko, G. E. (2015). Mechanisms of apoptosis. Biochemistry (Mosc.) 80, 1393-1405. doi: 10.1134/S0006297915110012

Sever, M. S., Vanholder, R., and Lameire, N. (2006). Management of crushrelated injuries after disasters. N. Engl. J. Med. 354, 1052-1063. doi: 10.1056/ NEJMra054329

Shi, Y., Xu, L., Tang, J., Fang, L., Ma, S., Ma, X., et al. (2017). Inhibition of HDAC6 protects against rhabdomyolysis-induced acute kidney injury. Am. J. Physiol. Renal Physiol. 312, F502-F515. doi: 10.1152/ajprenal.00546.2016

Su, Z., Yang, Z., Xu, Y., Chen, Y., and Yu, Q. (2015). Apoptosis, autophagy, necroptosis, and cancer metastasis. Mol Cancer 14:48. doi: 10.1186/s12943-0150321-5

Tang, J., Yan, Y., Zhao, T. C., Gong, R., Bayliss, G., Yan, H., et al. (2014). Class I HDAC activity is required for renal protection and regeneration after acute kidney injury. Am. J. Physiol. Renal Physiol. 307, F303-F316. doi: 10.1152/ ajprenal.00102.2014

Tang, W. X., Wu, W. H., Qiu, H. Y., Bo, H., and Huang, S. M. (2013). Amelioration of rhabdomyolysis-induced renal mitochondrial injury and apoptosis through suppression of Drp-1 translocation. J. Nephrol. 26, 1073-1082. doi: 10.5301/jn. 5000268

Taniguchi, M., and Yoshida, H. (2015). Endoplasmic reticulum stress in kidney function and disease. Curr. Opin. Nephrol. Hypertens. 24, 345-350. doi: 10.1097/ MNH.0000000000000141

Venkatachalam, M. A., Weinberg, J. M., Kriz, W., and Bidani, A. K. (2015). Failed tubule recovery, AKI-CKD transition, and kidney disease progression. J. Am. Soc. Nephrol. 26, 1765-1776. doi: 10.1681/ASN.2015010006

Walter, P., and Ron, D. (2011). The unfolded protein response: from stress pathway to homeostatic regulation. Science 334, 1081-1086. doi: 10.1126/ science. 1209038

Wang, M., and Kaufman, R. J. (2016). Protein misfolding in the endoplasmic reticulum as a conduit to human disease. Nature 529, 326-335. doi: 10.1038/ nature 17041

Yang, Z., Wang, T., Wang, F., Niu, T., Liu, Z., Chen, X., et al. (2016). Discovery of selective histone deacetylase 6 inhibitors using the quinazoline as the cap for the treatment of cancer. J. Med. Chem. 59, 1455-1470. doi: 10.1021/acs.jmedchem. $5 \mathrm{~b} 01342$

Youle, R. J., and Strasser, A. (2008). The BCL-2 protein family: opposing activities that mediate cell death. Nat. Rev. Mol. Cell Biol. 9, 47-59. doi: 10.1038/nrm2308

Conflict of Interest Statement: The authors declare that the research was conducted in the absence of any commercial or financial relationships that could be construed as a potential conflict of interest.

Copyright (C) 2018 Feng, Huang, Guo, Liang, Xiang, Lei, Shi, Li, Liu, Feng, Ma and Fu. This is an open-access article distributed under the terms of the Creative Commons Attribution License (CC BY). The use, distribution or reproduction in other forums is permitted, provided the original author(s) and the copyright owner are credited and that the original publication in this journal is cited, in accordance with accepted academic practice. No use, distribution or reproduction is permitted which does not comply with these terms. 